CLINICAL AND EXPERIMENTAL VACCINE RESEARCH

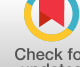

\title{
Hepatitis B vaccination and immunotherapies: an update
}

Clin Exp Vaccine Res 2020;9:1-7

https://doi.org/10.7774/cevr.2020.9.1.1

pISSN 2287-3651 • eISSN 2287-366X

\section{Cristina Stasi ${ }^{1,2}$, Caterina Silvestri ${ }^{1 *}$ Fabio Voller ${ }^{1 *}$ \\ 'Observatory of Epidemiology, Regional Health Agency of Tuscany, Florence; ${ }^{2}$ Interdepartmental Hepatology Center MASVE, Department of Experimental and Clinical Medicine, University of Florence, Florence, Italy \\ *These authors contributed equally to this work as senior authors.}

Received: December 4, 2019

Accepted: January 30, 2020

Corresponding author: Cristina Stasi, MD, PhD Observatory of Epidemiology, Regional Health Agency of Tuscany, 50141 Florence, Italy Tel: +39-055-4624385, Fax: +39-055-4624330

E-mail: cristina.stasi@gmail.com

No potential conflict of interest relevant to this article was reported.

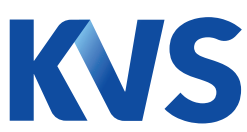

KOREA N

VACCINE

SOC IET Y

(C) Korean Vaccine Society.

This is an Open Access article distributed under the terms of the Creative Commons Attribution Non-Commercial License (https://creativecommons.org/licenses/ by-nc/4.0) which permits unrestricted non-commercial use, distribution, and reproduction in any medium, provided the original work is properly cited.
World Health Organization (WHO) estimates that 257 million people were living with chronic hepatitis B virus (HBV) infection. Highest HBV prevalence was found in the WHO Western Pacific Region (6.2\%) and in the WHO African Region (6.1\%). The HBV vaccine is the best protection against chronic HBV infection and its complications. Globally, routine infant immunization against HBV has increased with an estimated coverage of $84 \%$ in 2017. Nevertheless, in many countries further efforts are needed to implement this coverage and ensure national immunization programs for people at major risk for HBV infection. Therapeutic vaccination in chronic HBV infected patients can cause anti-HBV immune responses able to remove and/or cure infected hepatocytes. It shows promising results in murine model and human trials, but these results need to be consolidated by further multicenter clinical studies. In particular, the efficacy of therapeutic vaccine seems to improve by combination therapies.

Keywords: Hepatitis B virus infection, Vaccination, Therapeutic vaccine, Chronic hepatitis B

\section{Introduction}

In 2015, World Health Organization (WHO) estimates that 257 million people were living with chronic hepatitis B virus (HBV) infection (defined as hepatitis B surface antigen [HBsAg] positive) [1].

The highest prevalence of HBV infected population was found in the WHO Western Pacific Region (6.2\%) and the WHO African Region (6.1\%). The HBV prevalence was estimated of 3.3\% in the WHO Eastern Mediterranean Region, 2.0\% in the WHO SouthEast Asia Region and $1.6 \%$ in the WHO European Region. The lowest prevalence of HBV infected population (0.7\%) was found in the WHO Region of the Americas [1].

HBV infection is transmitted through contact with the blood or other bodily fluids of an infected person. Unsafe sex could put people at risk, as could getting a tattoo, piercing or manicure/pedicure in places with inadequate hygienic standards of utensils such as clippers and scissors. The HBV infection can cause serious health problems such as liver cancer, cirrhosis and liver failure resulting in death [2].

In 2017, the European Union and European Economic Area Member States reported 26.907 cases of HBV infection, of whom $9 \%$ were reported as acute, $58 \%$ as chronic, $32 \%$ as 'unknown' and $1 \%$ could not be classified [3]. In the WHO European Region an estimated 13.3 million people live with chronic HBV infection (1.8\% of adults) [4]. 
The people at higher risk for contracting infection are people who frequently require blood or blood products, dialysis patients and recipients of solid organ transplantations; people who inject drugs; inmates; household and sexual contacts of people with chronic HBV infection; people with multiple sexual partners; healthcare workers; travelers in endemic areas who have not completed their HBV vaccination. All of these groups should be vaccinated [1].

Several studies showed that the prevalence of HBV infection in prisoners ranged from $1.4 \%$ to $23.5 \%$. In fact, the highest prevalence of HBsAg was found in prisoners of West and Central African (23.5\%). High levels of chronic HBV infection have also been reported in Eastern and Southern Africa (5.7\%) and in Eastern Europe and Central Asia (10.4\%). The lowest prevalence was found in North America (1.4\%) [5].

The results of an Italian study [6], involving a total of 57 detention facilities, showed a HBV prevalence of $2.0 \%$. This prevalence was calculated on 15,751 inmates enrolled in this study, out of 17,086 inmates. For this study was designed a specific clinical record and all diagnoses were considered according to the International Classification of Diseases, Ninth Revision, Clinical Modification. The study showed that the prevalence of patients with chronic HBV infection is probably underestimated by the National Health Service, compared to that emerged from seroprevalence studies.

A cross-sectional screening study was conducted in Italy through the evaluation of serum markers for HBV infection (presence of HBsAg) in prison. The study identified $4.4 \%$ of HBsAg-positive subjects, of whom about $35 \%$ of foreigners [7]. Geue et al. [8] in a systematic review evaluated 15 studies concerning HBV screening on 2,284 initially considered. The authors found the dissimilarity between the different population groups examined, in particular some populations studied in the past (such as the general population) should not be screened in the future as the screening results not cost-effective. On the contrary, existing evidence suggests that screening activity in migrant populations could be a good cost-effective strategy. This result does not show changes based on the use of different economic models adopted, the evaluation of the qualityadjusted life years, the years of life gained, the number of cases detected, and the number of infections avoided.

Based on the clinical and public health relevance of HBV diffusion, the review will examine and discuss the new important strategies of HBV prevention and control by vaccination and the innovative vaccine therapy in chronic HBV patients.

\section{The anti-hepatitis B virus vaccination}

The vaccine against hepatitis $B$ is the best protection against chronic HBV infection and its complications, and it is included in routine childhood vaccinations in many countries. The vaccine against HBV has been available since 1982 and became widely available after the year 2000, therefore the adults of today may not have been vaccinated as children. The vaccine is extremely effective, and three doses give immunity for at least 20 years. The vaccine has greatly reduced the prevalence of HBV and the socioeconomic impact in industrialized countries.

WHO recommends that all infants receive the vaccine against $\mathrm{HBV}$ as soon as possible after birth, preferably within 24 hours. Routine infant immunization against HBV has increased globally with an estimated coverage (third dose) of $84 \%$ in 2017. The low prevalence of chronic HBV infection in children under 5 years of age, estimated at $1.3 \%$ in 2015, can be attributed to the widely use of vaccine against HBV [1].

In Europe routine childhood vaccination is included in 20 (74\%) of the 27 countries (Belgium, Bulgaria, Czech Republic, Cyprus, Estonia, France, Germany, Hungary, Italy, Malta, Latvia, Luxembourg, Portugal, Romania, Slovakia, Slovenia, Spain, Poland). In 12 (60\%) of these countries, namely Belgium, Cyprus, Estonia, France, Germany, Italy, Malta, Latvia, Romania, Poland, and Luxembourg, vaccination is planned not only in childhood but also in older children and in adolescents. Six countries have not yet introduced universal immunization against hepatitis B: Denmark, Finland, Iceland, Norway, Sweden, and United Kingdom. They have very low endemicity and consider hepatitis B to be a limited public health problem, thus not justifying additional expense [9].

In Italy vaccination, starting in 1991, is planned for all newborns who must undergo mandatory vaccination. Until 2003 (12 years after the law came into effect), adolescents were also vaccinated during the 12th year (the population born after 1979 received anti-HBV vaccination at the age of 12 years starting from 1991) [10].

\section{Vaccination for people at higher risk for contracting hepatitis B virus infection}

The Centers for Disease Control and Prevention highlighted the importance of blood screening for $\mathrm{HBV}$ and subsequent anti-HBV vaccination in the prison population for all people who received a medical evaluation in prison, unless they have 
of a proof of completion of the vaccination series or serological tests of immunity from infection [11]. Although vaccination against $\mathrm{HBV}$ has been recommended in prisoners since 1982, when the vaccine was made available, only a few countries usually vaccinate prisoners [7]. In Italy, a ministerial decree in October 1991 recommended vaccination against HBV in people at risk, including prisoners. Vaccination is currently recommended at months 0,1 , and 2 and again in month 6 .

For those who are homeless, there is an accelerated calendar for immunization against $\operatorname{HBV}(0,7$, and 21 days $)$ with a booster at 12 months, which results in a higher level of completion and seroconversion rates than traditional programs [12].

One of the main reasons for why inmates are not vaccinated is the lack of adherence to the screening program by prisoners. The reasons for non-compliance are probably attributable to both personal and institutional aspects such as lack of knowledge and awareness (perceived risk) regarding viral hepatitis and transmission routes, lack of motivation and/or awareness of the procedure, the fear of stigma by prison staff and colleagues, but also the lack of proactive strategies on the part of the staff and, not least, the lack of continuity of care after being released from prison.

Van Herck et al. [13] have shown that an accelerated program $(0,1,2$, and 12 months) or a super-accelerated program $(0,7,21$ days, and after 12 months) can lead to a faster response and increase in levels of anti-hepatitis B surface (HBs) antibodies $\geq 10 \mathrm{IU} / \mathrm{L}$. For the rapid seroconversion and the immediate protection in the short term, it is necessary to use the most accelerated program for groups at major risk of infection. Since no long-term protection data have been reported for these more accelerated programs, a fourth dose per month is still needed 12. Stasi et al. [14] evaluated 1,075 subjects screened for HBV serum markers, $67.9 \%$ were susceptible to infection and needed to be vaccinated, of these $82 \%$ agreed to be vaccinated. Five hundred and fifty-five inmates (95.1\%) received the first vaccine dose, and 404 (83\%) underwent the third dose at day 21 .

Almasio et al. [12] recommended vaccination against HBV in immigrants belonging to the following categories: coming from highly endemic areas, drug addiction, non-immunized prisoners, partner of an infected person, and patients with chronic liver disease not related to HBV. In particular, for all these groups an accelerated vaccination should be offered to allow a sufficiently high compliance rate and avoid dropouts.

Wright et al. [15] highlighted for homeless subjects the im- portance of an accelerated vaccination schedule $(0,7,21$ days $)$ with a booster at 12 months that results in higher completion rates and similar seroconversion rates compared to traditional schedules.

Wouters et al. [16] studied a highly mobile at-risk population of commercial sex workers in Belgium, and demonstrated that a 0-, 1-, 4-month schedule is easier to offer and confer equal protection within a shorter period of time. Mangen et al. [17] evaluated the cost-effectiveness of the decentralized targeted vaccination program against HBV for behavioral high-risk groups conducted by regional public health services in the Netherlands since November 1, 2002. Target groups for free vaccination were men having sex with men, commercial sex workers, and hard drug users. Heterosexuals with a high partner change rate were also included until November 1,2007 . The authors concluded that HBV-vaccination program is a cost-effective intervention in certain unvaccinated high-risk adults, and was a good alternative to national immunization programs. In the Netherlands, HBV vaccination was only introduced in the national immunization programs in 2011.

The US Preventive Services Task Force made recommendations screening for $\mathrm{HBV}$ infection in pregnant women at their first prenatal visit [18].

In line with international guidelines on $\mathrm{HBV}$ vaccination in human immunodeficiency virus (HIV)-infected population $[19,20]$, a recent review by Catherine and Piroth [21] highly recommended vaccination against $\mathrm{HBV}$, based on currently available data regarding $\mathrm{HBV}$ vaccination in people living with HIV, according to their main characteristics and their vaccine and therapeutic background. Response to vaccination is defined by a seroconversion with anti-HBs antibodies $>10 \mathrm{IU} / \mathrm{mL}$. In particular, double-dose rescue vaccination with at least three doses appears to induce better immunization against HBV in HIV-infected populations and an annual supervision of anti-HBs antibodies titers are recommended to monitor when new boosters are required.

\section{Beyond the current therapy for chronic hepatitis B virus infection}

The WHO guidelines for HBV prevention, care and treatment recommended treatment in all adults, adolescents, and children with chronic HBV infection with compensated or decompensated cirrhosis regardless of alanine aminotransferase (ALT) levels, hepatitis B e antigen (HBeAg) status, or HBV 
DNA levels [22]. Treatment is recommended for adults with chronic HBV infection who do not have clinical evidence of cirrhosis, but who are over the age of 30 years and have persistently abnormal ALT levels and high level of HBV replication (HBV DNA > 20,000 IU/mL), regardless of HBeAg status. These guidelines are based on a public health approach and they considered feasibility and effectiveness in countries with limited resources.

The current potent antiviral agents such as tenofovir and entecavir cause minimal risk of resistance, the viral suppression is over $99 \%$ and present very high rate of tolerability. Several lines of evidence suggest that long-term complete suppression of HBV replication by nucleosides/nucleotides (NUC) results in improved long-term outcomes such as the reduced risk of developing liver cirrhosis, hepatocellular insufficiency and hepatocellular carcinoma $[23,24]$ and histologically proven regression of liver fibrosis [25]. The disadvantages include the unlimited duration of treatment, the low rate of loss of HBsAg and the seroconversion to anti-HBs. Moreover, NUCs are still not able to eradicate the cccDNA.

HBV drugs are also currently being trialled, which induce persistent suppression of HBV-DNA, HBsAg negativity and eradication of cccDNA. Interferon induces both the immunomodulation and suppression of cccDNA transcription, but antiviral drugs target different steps of the HBV life cycle are also in development. These drugs are being tested which target the cccDNA, including inhibitors of the formation of cccDNA, transcription inhibitors and drugs that disrupt or degrade the cccDNA [26]. Other drugs still undergoing testing include translocation inhibitors of the virus in the cytoplasm or assembly of the nucleocapsid to DNA [27], or inhibitors of the release of HBsAg [28].

Finally, new immunomodulators are in a more advanced trial stage. Among these are HBV-specific immunomodulators and the anti-programmed cell death protein 1 and therapeutic vaccines which boosts the immune response.

\section{Vaccine therapy for chronic hepatitis B virus infection}

A complex interaction between virus and host causes HBV persistence with suboptimal immune responses, such as malfunctioning of cell-mediated immunity and dendritic cell and imbalance of cytokine production [29].

The therapeutic vaccination in chronic HBV infected pa-

tients can cause anti-HBV immune responses to remove and/ or cure infected hepatocytes without host cell damage, with subsequent prevention of viral spread to new hepatocytes and long-term viral control [30]. These approaches for stimulating T-cell responses with therapeutic vaccination include the use of different vaccination doses and frequencies and prime-boost; the use of DNA or peptide vaccines, vector or cell-based vaccines, and finally the use of a combination of core, $\mathrm{X}$ and polymerase antigens in addition to $\mathrm{HBsAg}[30,31]$.

One of the firsts clinical study on HBV vaccine therapy was carried out on 32 consecutive chronic HBsAg carriers with chronic hepatitis and detectable HBV DNA received three standard doses of the GenHevac B vaccine at one month intervals. Six months after the first dose, 12 patients (37.5\%) had undetectable HBV DNA while three others showed significant decrease in HBV DNA titers. Eight of these 15 responders received a standard course of $\alpha$-interferon and all still had undetectable HBV replication. Among 17 non responders to vaccine, 13 received $\alpha$-interferon, and only three stopped HBV replication. In conclusion, 53\% of HBV patients achieved undetectable HBV DNA [32]. Recently, Lai et al. [33] enrolled 20 chronic HBV infected e antigen-negative patients, with HBsAg $<1,000 \mathrm{IU} / \mathrm{mL}$. Seven vaccine doses were administered every 8 weeks and HBsAg levels and anti-HBs were longitudinally monitored until 48 weeks post-vaccination. In these patients, the vaccination resulted in significant HBsAg decline.

Bian et al. [34] investigated whether preS1-polypeptide vaccination is a potential treatment for chronic HBV infected patients. They found that the preS1 domain of L-HBsAg presents strong immunogenicity for both B-cell and T-cell responses. Moreover, the anti-preS1 induced by preS1-polypeptide cleared HBV DNA in carrier mice and blocked HBV infection/reinfection to hepatocytes, suggesting that the subsequent vaccination with HBsAg could induce anti-HBs seroconversion in HBV carrier mice.

In a phase I clinical trial, Zoulim et al. [35] studied safety, immunogenicity and efficacy of TG1050 in chronic HBV infected patients. TG1050 is an adenovirus 5-based vaccine that expresses HBV polymerase and domains of core and surface antigen. This study included two sequential phases: one single dose cohort and one multiple doses cohort. TG1050 was well tolerated in both cohorts and in association with NUC induced HBV-specific cellular immune response.

In a phase II clinical trial, Boni et al. [36] assessed the efficacy and safety of GS-4774 in viremic HBV infected patients. All of these patients received tenofovir disoproxil fumarate with or without GS-4774. GS-4774 is a yeast-based therapeu- 
Cristina Stasi et al $\bullet$ Hepatitis B vaccination and immunotherapies

tic vaccine containing.

HBV S, $X$, and core proteins. In murine model and cells from chronic HBV infected patients, GS-4774 induced interferon$\gamma$-producing CD4+ and CD8+ T cells [37]. Boni et al. [36] found that vaccination can increase production of interferon- $\gamma$, tumor necrosis factor, and interleukin 2 by CD8p $\mathrm{T}$ cells exposed to antigenic peptides, with little effect on CD4p T cells and they concluded that GS-4774 might be used in association with other antiviral treatment to boost the immune response.

In phase III clinical trial, Al Mahtab et al. [38] studied the efficacy of a therapeutic vaccine (NASVAC) containing both HBsAg and hepatitis B core antigen versus pegylated interferon in a total of 160 chronic HBV infected patients. In this study, the viral load significantly decreased in NAVASC group compared to pegylated interferon-group at 24 weeks of follow-up. Clearance rate of $\mathrm{HBeAg}$ was also more frequent in NASVAC group compared to pegylated interferon, but a slight progression into cirrhosis was found in NASVAC group compared to pegylated-IFN group. Brillanti et al. [39] in a pilot study randomly assigned five patients with HBeAg-negative chronic HBV infection to receive HBV vaccine therapy: three doses, 1 month apart, of $40 \mathrm{mcg}$ yeast derived recombinant hepatitis B vaccine (HBVAXPro), while five patients continued nucleo$\mathrm{s}(\mathrm{t})$ ide analog therapy without vaccination. HBsAg and HBV DNA status was assessed 6 months afterwards. In HBVAXPro group the vaccine therapy enhanced HBsAg loss and anti-HBs seroconversion.

\section{Conclusion}

The HBV vaccine is the best protection against chronic HBV infection and its complications. Globally, routine infant immunization against HBV has increased with an estimated coverage of $84 \%$ in 2017 , but further efforts are needed to implement this coverage in many countries and ensure national strategies for people at major risk of contracting HBV infection. Severe lines of evidences indicate that therapeutic vaccine has promising results in murine model and human trials, but these results need to be consolidated by further multicenter research clinical studies. In particular, the efficacy of therapeutic vaccine able to enhance T-cell responsiveness seems to improve by combination therapies.

\section{ORCID}

Cristina Stasi https://orcid.org/0000-0002-9146-9968
Caterina Silvestri https://orcid.org/0000-0002-1577-2869

Fabio Voller https://orcid.org/0000-0002-1063-415X

\section{References}

1. World Health Organization. Hepatitis B [Internet]. Geneva: World Health Organization; 2019 [cited 2019 Jul 18]. Available from: https://www.who.int/news-room/factsheets/detail/hepatitis-b.

2. World Health Organization Regional officer for Europe. Learn the ABCs of hepatitis before you leave on holiday [Internet]. Copenhagen: World Health Organization Regional officer for Europe; 2019 [cited 2019 Jul 23]. Available from: http://www.euro.who.int/en/health-topics/ communicable-diseases/hepatitis/news/news/2019/7/ learn-the-abcs-of-hepatitis-before-you-leave-on-holiday.

3. European Centre for Disease Prevention and Control. Hepatitis B: annual epidemiological report for 2017 [Internet]. Solna: European Centre for Disease Prevention and Control; 2019 [cited 2019 Jun 17]. Available from: https://ecdc. europa.eu/en/publications-data/hepatitis-b-annual-epidemiological-report-2017.

4. World Health Organization. Data and statistics [Internet]. Copenhagen: World Health Organization Regional officer for Europe; 2019 [cited 2019 Jul 23]. Available from: http:// www.euro.who.int/en/health-topics/communicable-diseases/hepatitis/data-and-statistics.

5. Dolan K, Wirtz AL, Moazen B, et al. Global burden of HIV, viral hepatitis, and tuberculosis in prisoners and detainees. Lancet 2016;388:1089-102.

6. Stasi C, Silvestri C, Fanti E, Di Fiandra T, Voller F. Prevalence and features of chronic viral hepatitis and HIV coinfection in Italian prisons. Eur J Intern Med 2016;34:e21-2.

7. Sagnelli E, Starnini G, Sagnelli C, et al. Blood born viral infections, sexually transmitted diseases and latent tuberculosis in Italian prisons: a preliminary report of a large multicenter study. Eur Rev Med Pharmacol Sci 2012;16: 2142-6.

8. Geue C, Wu O, Xin Y, et al. Cost-effectiveness of HBV and HCV screening strategies: a systematic review of existing modelling techniques. PLoS One 2015;10:e0145022.

9. World Health Organization. 2019. Documenting the impact of hepatitis B immunization: best practices for conducting a serosurvey [Internet]. Geneva: World Health Organization; 2019 [cited 2019 Jul 23]. Available from: https: //apps.who.int/iris/bitstream/handle/10665/70808/WHO_ 
IVB_11.08_eng.pdf?sequence=1.

10. Ministry of Health. Vaccination for hepatitis B: clarifications to DM 20/11/2000 [Internet]. Rome: Ministry of Health; 2000 [cited 2019 Jul 23]. Available from: http://www.trovanorme.salute.gov.it/normsan-pdf/0000/28940_1.pdf.

11. Centers for Disease Control and Prevention. Prevention and control of infections with hepatitis viruses in correctional settings [Internet]. Atlanta, GA: Centers for Disease Control and Prevention; 2003 [cited 2019 Jul 23]. Available from: https://www.cdc.gov/Mmwr/preview/mmwrhtml/ rr5201al.htm.

12. Almasio PL, Babudieri S, Barbarini G, et al. Recommendations for the prevention, diagnosis, and treatment of chronic hepatitis $\mathrm{B}$ and $\mathrm{C}$ in special population groups (migrants, intravenous drug users and prison inmates). Dig Liver Dis 2011;43:589-95.

13. Van Herck K, Leuridan E, van Damme P. Schedules for hepatitis B vaccination of risk groups: balancing immunogenicity and compliance. Sex Transm Infect 2007;83: 426-32.

14. Stasi C, Monnini M, Cellesi V, et al. Screening for hepatitis $B$ virus and accelerated vaccination schedule in prison: a pilot multicenter study. Vaccine 2019;37:1412-7.

15. Wright NM, Campbell TL, Tompkins CN. Comparison of conventional and accelerated hepatitis B immunisation schedules for homeless drug users. Commun Dis Public Health 2002;5:324-6.

16. Wouters K, Leuridan E, van Herck K, et al. Compliance and immunogenicity of two hepatitis B vaccination schedules in sex workers in Belgium. Vaccine 2007;25:1893-900.

17. Mangen MJ, Stibbe H, Urbanus A, et al. Targeted outreach hepatitis B vaccination program in high-risk adults: the fundamental challenge of the last mile. Vaccine 2017;35: 3215-21.

18. US Preventive Services Task Force, Owens DK, Davidson $\mathrm{KW}$, et al. Screening for hepatitis B virus infection in pregnant women: US Preventive Services Task Force reaffirmation recommendation statement. JAMA 2019;322:34954.

19. AIDSinfo. Guidelines for prevention and treatment of opportunistic infections in HIV-infected adults and adolescents [Internet]. Rockville, MD: AIDSinfo; 2018 [cited 2019 Oct 1]. Available from: https://aidsinfo.nih.gov/guidelines/ $\mathrm{html} / 4$ /adultand-adolescent-oiprevention-and-treatmentguidelines $/ 344 / \mathrm{hbv}$.

20. Wilkins E, Nelson M, Agarwal K, et al. British HIV Associa- tion guidelines for the management of hepatitis viruses in adults infected with HIV 2013. HIV Med 2013;14 Suppl 4:1-71.

21. Catherine FX, Piroth L. Hepatitis B virus vaccination in HIV-infected people: a review. Hum Vaccin Immunother 2017;13:1-10.

22. World Health Organization. Guidelines for the prevention, care and treatment of persons with chronic hepatitis B infection [Internet]. Geneva: World Health Organization; 2015 [cited 2019 Mar 20]. Available from: http://apps.who. int/iris/bitstream/10665/154590/1/9789241549059eng. pdf?ua $=1[7]$.

23. Tang H, Griffin J, Innaimo S, Lehman-Mckeeman L, Llamoso C. The discovery and development of a potent antiviral drug, Entecavir, for the treatment of chronic hepatitis B. J Clin Transl Hepatol 2013;1:51-8.

24. Tan ZM, Sun BC. Effects of antiviral therapy on preventing liver tumorigenesis and hepatocellular carcinoma recurrence. World J Gastroenterol 2013;19:8895-901.

25. Bourliere M, Kahloun A, Gascou-Tessonnier G. Analogs and fibrosis regression in hepatitis B. Gastroenterol Clin Biol 2009;33:923-9.

26. Cai D, Mills C, Yu W, et al. Identification of disubstituted sulfonamide compounds as specific inhibitors of hepatitis $B$ virus covalently closed circular DNA formation. Antimicrob Agents Chemother 2012;56:4277-88.

27. Zoulim F, Yogaratnam JZ, Vandenbossche JJ, et al. Safety, pharmakokinetics and antiviral activity of novel capsid assembly modulator (CAM) JNJ-56136379 (JNJ-6379) in treatmentnaive chronic hepatitis B (CHB) patients without cirrhosis. J Hepatol 2018;68:S102.

28. Quinet J, Jamard C, Vaillant A, Cova L. Achievement of surface antigen clearance in the liver by combination therapy with REP 2139-Ca and nucleoside analogues against chronic hepatitis B. J Hepatol 2016;64(Suppl 2):S285.

29. Tseng TC, Huang LR. Immunopathogenesis of hepatitis B virus. J Infect Dis 2017;216(suppl_8):S765-70.

30. Liang TJ, Block TM, McMahon BJ, et al. Present and future therapies of hepatitis B: from discovery to cure. Hepatology 2015;62:1893-908.

31. Lobaina Y, Michel ML. Chronic hepatitis B: immunological profile and current therapeutic vaccines in clinical trials. Vaccine 2017;35:2308-14.

32. Pol S. Immunotherapy of chronic hepatitis B by anti HBV vaccine. Biomed Pharmacother 1995;49:105-9.

33. Lai MW, Hsu CW, Lin CL, et al. Multiple doses of hepatitis 
B recombinant vaccine for chronic hepatitis B patients with low surface antigen levels: a pilot study. Hepatol Int 2018;12:456-64.

34. Bian Y, Zhang Z, Sun Z, et al. Vaccines targeting preS1 domain overcome immune tolerance in hepatitis B virus carrier mice. Hepatology 2017;66:1067-82.

35. Zoulim F, Fournier C, Habersetzer F, et al. Safety and immunogenicity of the therapeutic vaccine TG1050 in chronic hepatitis B patients: a phase $1 \mathrm{~b}$ placebo-controlled trial. Hum Vaccin Immunother 2019:1-12.

36. Boni C, Janssen HL, Rossi M, et al. Combined GS-4774 and tenofovir therapy can improve HBV-specific T-cell responses in patients with chronic hepatitis. Gastroenterology 2019;157:227-41.
37. King TH, Kemmler CB, Guo Z, et al. A whole recombinant yeast-based therapeutic vaccine elicits $\mathrm{HBV}$ X, S and Core specific $\mathrm{T}$ cells in mice and activates human $\mathrm{T}$ cells recognizing epitopes linked to viral clearance. PLoS One 2014; 9:e101904.

38. Al Mahtab M, Akbar SM, Aguilar JC, et al. Treatment of chronic hepatitis $B$ naive patients with a therapeutic vaccine containing $\mathrm{HBs}$ and $\mathrm{HBc}$ antigens (a randomized, open and treatment controlled phase III clinical trial). PLoS One 2018;13:e0201236.

39. Brillanti S, Laterza L, Cecinato P, Bazzoli F. Loss and seroconversion of hepatitis B surface antigen after vaccine therapy in chronic hepatitis B patients treated with nucleoside/ nucleotide analogues. Hipatol Int 2013;7:S169-70. 